\title{
Cursos MOOC: factores que disminuyen el abandono en los participantes
}

\section{(MOOCs: factors that decrease desertion in students)}

\author{
Marco Yamba-Yugsi ${ }^{1}$, Sergio Luján-Mora²
}

\begin{abstract}
Resumen:
Estamos viviendo constantes cambios tecnológicos y las necesidades de aprendizaje y capacitación son cada vez mayores. Uno de esos cambios son los cursos masivos abiertos en línea (MOOC). Como este es un nuevo tipo de curso en línea en nuestro entorno y el índice de abandono es alto, es imprescindible su análisis para conocer las razones de este y lograr disminuir la deserción de usuarios en cursos MOOC. Para ello, se propone conocer diferentes factores del problema, como por ejemplo la experiencia previa de los usuarios en la realización de cursos MOOC, el nivel de satisfacción en la interacción con las plataformas que alojan cursos MOOC y la satisfacción en general al finalizar un curso. Además, el conocer el perfil de los usuarios facilitará que los futuros cursos se adapten a ellos, aunque conlleve incrementar el equipo de trabajo. Con el fin de que estos se sientan acompañados, con ánimo de seguir el curso y que posean una nueva experiencia de aprendizaje.
\end{abstract}

Palabras clave: Cursos MOOC; plataformas virtuales; proyecto Mineduc; abandono.

\begin{abstract}
:
We are experiencing constant technological change, which implies that learning and training requirements have been increased, they are increasingly high. One of these changes are Massive Open Online Courses (MOOC). As this is a new technology in our environment, the amount of desertion is high, it is essential to the analysis, to know the reasons of the high amounts of desertion and reduce the dropout of users in MOOC courses. For that, it is proposed to meet the different factors of the problem, for example, previous experience of users in making the MOOC courses, the level of satisfaction in the interaction with platforms that have courses MOOC and satisfaction by the end of a course. Additionally, knowing the user's profile will make it easier for future courses suit them, although entails increasing the team and tailor a course to users, for the purpose of that they feel supported, intending to follow course and that they possess a new learning experience.
\end{abstract}

Keywords: MOOC courses; virtual platforms; Mineduc project; abandonment.

\footnotetext{
1 Instituto de Altos Estudios Nacionales (CEC-IAEN), Quito - Ecuador (myamba@cec-iaen.edu.ec).

2 Universidad de Alicante, Alicante - España (sergio.lujan@ua.es).
} 


\section{Introducción}

\subsection{Un poco de historia acerca de los MOOC}

El año 2012 el periódico The New York Times declaró como el año de los MOOC (Pappano, 2012) y un sinnúmero de empresas empezaron sus emprendimientos en desarrollo de plataformas MOOC, siendo las principales actualmente Udacity, Coursera, Open edX, Udemy y Miríada X.

No obstante, la historia de los MOOC empezó cuatro años antes, en agosto de 2008, con el proyecto CCK08 (curso "Connectivism and Connective Knowledge") dictado por George Siemens y Stephen Downes (Lazo \& Contreras, 2013). En este curso de 12 semanas de duración se inscribieron aproximadamente unos 2.300 estudiantes de diferentes partes del mundo. Evidentemente, no tuvo el mismo éxito que los MOOC actuales, pero abrió el camino a los que hoy en día se dan (Pernías \& Luján-Mora, 2013). El mismo año fue acuñado el término MOOC por Dave Cormier y Brian Alexander, haciendo alusión a los Massively Multiplayer Online Game (Cabero, Llorente, \& Vázquez, 2014).

Posteriormente, "George Siemens, Stephen Downes y Dave Cormier organizaron otros cursos MOOC similares (CCK09, CCK11, CCK12, Future of Education, PLENK, LAK11, LAK12, Change11, Critical Literacies), que en total acumularon más de 20.000 inscripciones entre todos ellos" (Pernías \& Luján-Mora, 2013).

Los MOOC captan el total interés y la atención de los académicos y público general, puesto que se relacionan con la pedagogía eficiente y principios de diseño, derechos de autor y la garantía de calidad en la educación a distancia y en línea. Demuestran visiblemente ser una iniciativa eficaz para el aprendizaje cotidiano, pues ofrecen mucha información útil (Lazo \& Contreras, 2015).

Cada término de las siglas MOOC puede ser negociable. Es decir, no se tiene que cumplir con el significado de cada una de las letras de MOOC (Luján-Mora, 2013). Este puede ser en línea pero no abierto a todo público, o también abierto pero no tiene que ser totalmente gratis, como hacen las principales plataformas que cobran por el certificado acreditado por la universidad que da el curso.

\subsection{Importancia}

A partir del éxito del curso de Inteligencia Artificial impartido por la Universidad de Stanford, un buen número de las universidades se interesó por este tema (Haywood \& Macleod, 2014). Los MOOC emergieron como el canal ideal para instruirse cuando y donde uno quiera; tienen un gran 
potencial para proporcionar oportunidades para las personas de todo el mundo (Sanchez-Gordon \& Luján-Mora, 2014).

Un propósito es contribuir al aprendizaje inclusivo, ya que pueden brindar una experiencia para miles de usuarios de todas las condiciones y discapacidades al mismo tiempo (Sanchez-Gordon \& Luján-Mora, 2016). Con esta promesa los MOOC ayudarían a democratizar la educación, llegando a una educación inclusiva sin barreras.

Las plataformas MOOC ofrecen a los estudiantes la oportunidad de capacitarse y obtener certificados internacionales (Borrás, Martínez, \& Fidalgo, 2014). En estos cursos se imparten temas que son de interés general, para una región, un país o un sector en específico (Mailhes \& Raspa, 2015), dependiendo de la plataforma a la cual se registre el usuario. También dan la posibilidad de comprar el certificado de la universidad que ha dado el curso. Es decir, se usan como herramientas que ofrecen a los estudiantes la oportunidad de capacitarse y aumentar el conocimiento, de una forma bastante económica.

Los cursos MOOC pueden ayudar a las clases presenciales como un recurso adicional en algunas asignaturas (Luján-Mora, 2013) facilitando el quehacer tanto del docente como del estudiante, mediante contenidos de calidad que facilitan las mejores universidades del planeta.

\subsection{Abandono}

Una forma diferente de aprender hoy en día es a través de los MOOC, que se están integrando poco a poco al sistema educativo tradicional y a la vez, mejorando las oportunidades de aprendizaje. Sin embargo, el abandono de participantes en los MOOC es muy alto, como se puede evidenciar en el estudio realizado por la Universidad de Pennsylvania durante los años 2012 y 2013, en el proyecto "Penn GSE" donde dio como resultado que solo el $4 \%$ de los estudiantes concluía los MOOC; en este estudio se analizó un millón de usuarios de 16 cursos en la plataforma "Coursera" (Stein, 2013). Mientras que en 2015 solo un 15\% de quienes iniciaron un MOOC lo terminó (Jordan, 2015).

En la última década, los estudiantes han examinado el proceso de aprendizaje de diversas maneras, cada vez más digitales e interactivas (Cormier \& Siemens, 2010). La mayoría de estudiantes no están preparados, y el tener que realizar las tareas de una nueva manera aumenta considerablemente que estudiantes los abandonen.

Los entornos de aprendizaje desde sus inicios han presentado inconvenientes con el abandono de cursos por parte de sus estudiantes (MOOC-Maker, 2016). Los cursos MOOC no son la excepción y tienen un índice de abandono muy alto en la actualidad. 
Los MOOC se encuentran en una etapa de "depresión de desilusión" (Sanchez-Gordon \& LujánMora, 2016). El número deserciones en los cursos es la principal barrera por la cual han tenido opiniones negativas (Ramírez \& Carrillo, 2016); por tal motivo, es necesario que más personas concluyan cursos y de esta manera las opiniones negativas disminuyan.

\subsection{Contexto del Proyecto Mineduc}

El Proyecto Mineduc es un convenio de capacitación entre dos instituciones públicas de Ecuador. Fue firmado en noviembre de 2014 entre el Ministerio de Educación (Mineduc) y el Instituto de Altos Estudios Nacionales (IAEN), para capacitar a 5.000 docentes del Magisterio en el área de Educación General Básica (EGB). De este se encargaría el Centro de Educación Continua del IAEN (CEC-IAEN), al ejecutar cinco cursos ("Introducción a la plataforma", "Matemática", "Ciencias Naturales", "Lengua y Literatura" y "Ciencias Sociales"). El proyecto concluyó en su totalidad en abril de 2016.

Para capacitar a los docentes fue necesaria una plataforma de educación masiva, para lo cual el IAEN puso a disposición la que tenía desde 2013, llamada formaX (anteriormente llamada upeX) (Sanchez-Gordon, Calle-Jimenez, \& Lujan-Mora, 2015) para impartir cursos en línea, que fue construida bajo la tecnología de Open edX y es de uso libre y código abierto (edX Inc, 2016).

Los participantes provenientes de distintos lugares de Ecuador fueron escogidos por el Ministerio de Educación; ellos recibieron el curso totalmente en línea y la evaluación final fue presencial. El porcentaje de la nota en la plataforma mediante actividades realizadas fue de un $60 \%$ y el de la prueba final presencial de un 40\%; sumados los dos, el participante debía cumplir con un $70 \%$ como mínimo para pasar en cada materia. Los que pasaron los cuatro cursos (a excepción de "Introducción a la plataforma", que fue opcional) obtuvieron un certificado equivalente a 330 horas y podrían aplicar para el ascenso en el escalafón del Magisterio nacional.

En este artículo se presentan los dos primeros cursos, llevados a cabo desde el 10 de enero al 19 de septiembre de 2015. Estos se describen a continuación:

- "Introducción a la Plataforma". El objetivo principal del curso fue capacitar a los docentes de Educación General Básica sobre el uso de la plataforma, desde el 10 de enero hasta el 10 de febrero de 2015. Este curso constaba de cuatro módulos, compuestos de diferentes recursos que brinda la plataforma, como videos cortos, lecturas, foros y cuestionarios.

- “Matemática". Una vez concluido el primer curso se continuó con el de "Matemática", que se llevó a cabo desde el 8 de julio hasta el 19 de septiembre de 2015. Fue el más largo de todos y constó de 6 módulos. 
Según trabajos previos, la infraestructura inadecuada (MOOC-Maker, 2016), la incorrecta estructura del curso (Gomez \& De la Garza, 2016), la falta de conocimiento técnico (MOOCMaker, 2016) al iniciar el curso son los principales motivos del abandono de los MOOC.

El propósito de este artículo es dar a conocer los resultados encontrados en las encuestas, para que puedan ser aplicados en proyectos similares, o que sirvan como punto de partida para nuevas investigaciones. Así, nuevos usuarios terminarán cursos MOOC con éxito, basados en el nivel tecnológico que posean, contenidos y actividades interactivas.

El presente artículo aborda y explora varios temas: donde se empieza con la introducción, en la cual se describe la historia de los MOOC, importancia, abandono y el contexto del Proyecto Mineduc, en la cual se basó para elaborar este artículo. A continuación se explica el "Método", donde se revisan las herramientas tecnológicas de encuestas utilizadas, Encuestas realizadas, Mapeo de encuestas. En "Resultados" se presentan los datos de mayor relevancia para el estudio realizado en las dos encuestas a los 5.000 docentes, mediante descripciones gráficos y tablas. En "Discusión" se realiza una comparación de los datos obtenidos con estudios similares y para finalizar se detallan las conclusiones y recomendaciones del artículo presentado.

\section{Método}

Se plantearon objetivos de investigación, según los cuales se sacó estas preguntas de investigación: ¿cuál es el estado de ánimo, previo a participar en el curso MOOC?, ¿cuál es la principal motivación para concluir el curso?, ¿cuál es el nivel de satisfacción al concluir los dos cursos?

Las preguntas de investigación han sido divididas en las dos encuestas de forma sencilla y clara para que cualquier participante las pueda entender, mediante respuestas cerradas, ya sea de "Sí" o "No", o a su vez utilizando la "Escala de Likert", pues de esta forma se facilita la medición de los resultados, mediante la utilización de herramientas tecnológicas como Google Form³ y Typeform

Se realizó una encuesta de diagnóstico (ED) y otra encuesta de satisfacción (ES), al mismo tiempo que se impartían los dos MOOC dados en la plataforma formaX, de enero a septiembre de 2015, "Introducción a la plataforma" y "Matemática". En estos se midió principalmente la experiencia previa, el estado de ánimo y la satisfacción de los participantes, al concluir con el

\footnotetext{
${ }^{3}$ Formularios de Google: https://www.google.com/intl/es-419/forms/about/

${ }^{4}$ Formularios de Typeform: https://www.typeform.com/
} 
segundo curso de "Matemática". Así se quiso lograr que la mayor cantidad de usuarios terminase el curso.

Se realizó una encuesta de diagnóstico al iniciar el curso "Introducción a la Plataforma", mediante la que se deseaba conocer el perfil de los participantes: estado de ánimo, conocimiento informático y experiencia en los MOOC. Con los datos recopilados, el experto en contenidos y el diseñador instruccional debían organizar el siguiente curso "Matemática", a través de recursos, actividades y tareas en la plataforma MOOC. El seguimiento del curso en los foros y el servicio de soporte técnico son importantes para realizar cambios en tiempo real, cuando varios usuarios tengan inconvenientes con el uso de algún recurso en la plataforma.

Luego de haber culminado los dos cursos, fue necesario saber sobre la satisfacción de los participantes. Por tal motivo, se desarrolló una encuesta de satisfacción, de forma anónima y opcional, tal como se manifestó en el mensaje de participación.

\subsection{Herramientas de encuestas utilizadas}

Una vez seleccionadas las preguntas que irían en cada encuesta, se procedió a cargar la ED en Google Forms, que fue embebido dentro del curso "Introducción a la Plataforma", en la primera sección. En la ES en el curso de "Matemática", por preferencias visuales y de adaptabilidad, optamos por Typeform, que da un mejor resultado visual cuando se lo embebe en la plataforma.

\subsection{Encuestas realizadas}

Se realizaron dos encuestas: la ED al comenzar el curso de "Introducción a la Plataforma" y la ES al finalizar el curso de "Matemática". En las dos encuestas se colocó el siguiente mensaje: "La presente encuesta es anónima y opcional, con el fin de que se sienta en libertad de contestar de la mejor manera la encuesta".

Las principales preguntas utilizadas para la ED fueron las siguientes:

6. Razones por las que realizará este curso (señala la opción preferente).

7. Si en la pregunta anterior respondió "otro", indique la razón para realizar el curso.

8. ¿Qué expectativas caracterizan su participación en este curso?

- Motivación.

- Entusiasmo.

- Interés.

- Desconcierto.

- Miedo. 
Las principales preguntas utilizadas en la ES fueron las siguientes:

6. ¿Los medios utilizados (videos, foros, lecturas, etc.) estimularon y motivaron su participación en el curso?

8. ¿La forma de organizar el curso es adecuada?

9. ¿La metodología del curso ha fomentado la colaboración?

17. ¿La carga de trabajo del curso le pareció adecuada?

18. ¿Los cuestionarios de evaluación le han permitido evaluar su progreso en el curso?

19. ¿Los cuestionarios de evaluación tienen un grado de dificultad adecuado?

20. ¿La relación entre esfuerzo invertido y objetivos alcanzados le ha parecido adecuada?

21. ¿Cómo calificaría el apoyo del instructor durante el curso?

22. ¿Ha tenido algún tipo de problema a la hora de presentar un trabajo o un quiz, por este medio?

23. ¿Cómo calificaría la navegación en la plataforma?

\subsection{Mapeo de Encuestas}

Para una mejor comprensión de las preguntas distribuidas en las dos encuestas, es conveniente agruparlas de acuerdo a los objetivos, los cuales son:

- Medir el nivel de conocimiento tecnológico de los participantes en el curso MOOC.

- Conocer el estado de ánimo previo a participar en el curso MOOC.

- Medir la organización, colaboración y evaluación del curso.

\section{Medir el nivel de conocimiento tecnológico de los participantes en el curso MOOC}

Este objetivo tiene relación con las preguntas $(3,4,5,6$ y 10) de la ED y este, a la vez, tiene relación con las preguntas $(4,6,9,10,11$ y 12$)$ de la ES.

Para medir el nivel de conocimiento se dividieron las preguntas en las dos encuestas: la primera al inicio del curso "Introducción a la Plataforma", con el objetivo de saber la experiencia que tienían los participantes en cursos MOOC; en el segundo curso, "Matemática", se quiso conocer la satisfacción en la interacción con las herramientas de la plataforma. 


\section{Conocer el estado de ánimo previo a participar en el curso MOOC}

Este objetivo tiene relación con las preguntas (7, 8 y 9) de la ED y este, a la vez, tiene relación con la ES en la pregunta (13).

Para un correcto manejo del curso, no solo es necesario conocer acerca de la tecnología, sino también la actitud y la disposición emocional que el usuario tiene para participar en el curso. Si el usuario tiene una actitud positiva el curso tendrá más posibilidades de ser un éxito.

\section{Medir la organización colaboración y evaluación del curso}

Este objetivo tiene relación con las preguntas $(2,5,7,8,16,17,18,19,20,21$ y 22).

Las preguntas fueron planteadas al final del segundo curso, para conocer el grado de conformidad respecto a los recursos y actividades planteadas en el curso.

\section{Resultados}

En el curso de Matemática participaron 5.000 docentes y aprobaron 3.060, correspondientes a un $62,1 \%$. Un porcentaje superior a los que se tuvo en cursos MOOC durante 2015 que fue de un 15\% (Jordan, 2015).

En la ED participaron 367 personas, mientras que en la ES participaron 3.020 personas. La diferencia del número de participantes en las dos encuestas se debió a que el primer curso fue opcional y el segundo ya contaba para el certificado de 330 horas.

\section{Pregunta 6 (ED): Razones por las que realizará este curso (señala la opción preferente)}

La mayoría, el 82,6\% correspondiente a 303 personas, dijo que el curso lo realizó por "Aplicar en la actividad como profesor". Solo 4, correspondientes al 1,1\%, contestaron por ser por exigencia de la institución, como se puede observar a continuación en la Figura 1, en la que se muestran los porcentajes de las razones declaradas para realizar el curso. 


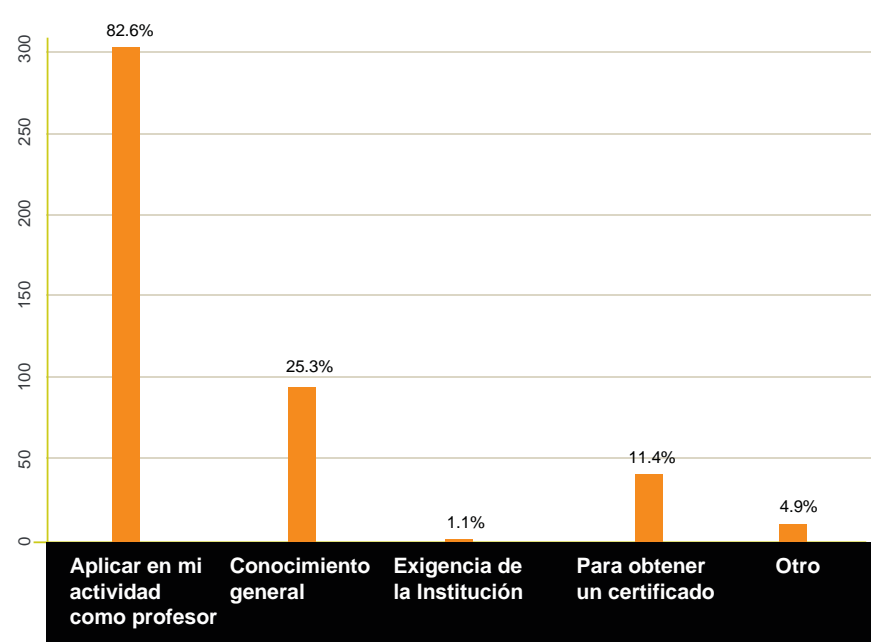

Figura 1. Razones por las que profesores realiza el curso "Introducción a la plataforma" en Ecuador, 2015.

\section{Pregunta 8 (ED): ¿Qué expectativas caracterizan su participación en este curso?}

Para conocer el estado de ánimo de los participantes en los cursos se realizaron, en la ED, tres preguntas con expectativas positivas (Motivación, Entusiasmo e Interés) y dos con expectativas negativas (Desconcierto y Miedo). Para evaluar se utilizó una escala de "nada", "poco", "medio", "mucho" y "no aplica", en la que los resultados han sido positivos, como se puede ver en la el Figura 2, donde se muestra el estado de ánimo de los usuarios traducido en porcentajes.

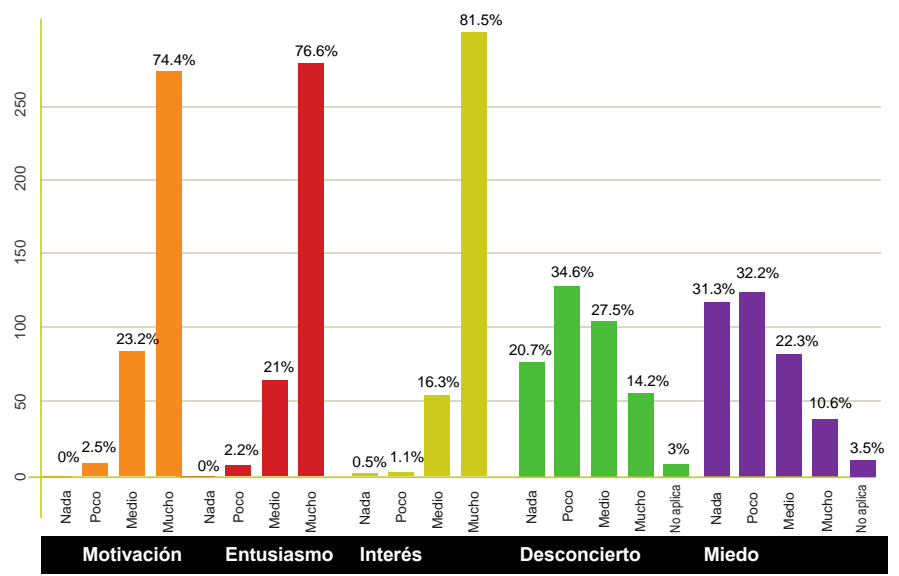

Figura 2. Estado de ánimo previo al inicio del curso "Introducción a la plataforma" en Ecuador, 2015.

En la segunda encuesta ES, se plantearon las preguntas de manera diferente. Para poder conocer los resultados se basó en la "Escala de Likert" con 5 niveles, donde 1 significa que el participante está "totalmente de acuerdo"; 2, "de acuerdo"; 3, "ni en acuerdo ni en desacuerdo"; 4, "en desacuerdo"; y 5, "totalmente en desacuerdo".

En esta encuesta participaron 3.020 personas y se obtuvieron resultados positivos en cuanto a nuestra pregunta de investigación: ¿cuál es el nivel de satisfacción al concluir los dos cursos?, que van desde un $40 \%$ a un $56 \%$ de estar "Totalmente de acuerdo", como se puede observar en 
la Tabla 1, que está relacionado con el número de participantes que aprobaron el módulo de Matemática correspondientes al $62,1 \%$.

Tabla 1: Porcentajes de satisfacción en el curso "Matemática” en Ecuador, 2015.

\begin{tabular}{|c|c|c|c|c|c|}
\hline & 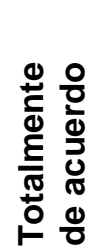 & 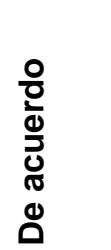 & 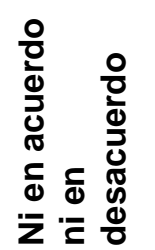 & 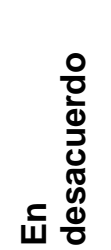 & 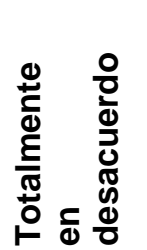 \\
\hline $\begin{array}{l}\text { Pregunta } 6(\mathrm{ES}) \text { : ¿Los medios utilizados (videos, foros, } \\
\text { lecturas, etc.) estimularon y motivaron su participación en } \\
\text { el curso? }\end{array}$ & $51 \%$ & $26 \%$ & $14 \%$ & $6 \%$ & $3 \%$ \\
\hline $\begin{array}{l}\text { Pregunta } 8 \text { (ES): ¿La forma de organizar el curso es } \\
\text { adecuada? }\end{array}$ & $48 \%$ & $27 \%$ & $15 \%$ & $6 \%$ & $5 \%$ \\
\hline $\begin{array}{l}\text { Pregunta } 9(E S) \text { : ¿La metodología del curso ha fomentado } \\
\text { la colaboración? }\end{array}$ & $47 \%$ & $29 \%$ & $15 \%$ & $5 \%$ & $4 \%$ \\
\hline $\begin{array}{l}\text { Pregunta } 17 \text { (ES): ¿La carga de trabajo del curso le } \\
\text { pareció adecuada? }\end{array}$ & $40 \%$ & $30 \%$ & $16 \%$ & $8 \%$ & $6 \%$ \\
\hline $\begin{array}{l}\text { Pregunta } 18(E S) \text { : ¿Los cuestionarios de evaluación le han } \\
\text { permitido evaluar su progreso en el curso? }\end{array}$ & $56 \%$ & $26 \%$ & $12 \%$ & $3 \%$ & $3 \%$ \\
\hline $\begin{array}{l}\text { Pregunta } 21 \text { (ES): ¿Cómo calificaría el apoyo del instructor } \\
\text { durante el curso? }\end{array}$ & $40 \%$ & $31 \%$ & $16 \%$ & $7 \%$ & $6 \%$ \\
\hline $\begin{array}{l}\text { Pregunta } 23 \text { (ES): ¿Cómo calificaría la navegación en la } \\
\text { plataforma? }\end{array}$ & $55 \%$ & $29 \%$ & $10 \%$ & $3 \%$ & $2 \%$ \\
\hline
\end{tabular}

Pregunta 19 (ES): ¿Los cuestionarios de evaluación tienen un grado de dificultad adecuado?

De 3.020 encuestas, 2.346 respondieron que los cuestionarios tienen un grado de dificultad adecuado y esto corresponde al $78 \%$, mientras que 674 , que corresponden al $22 \%$, no está de acuerdo con la dificultad dada a los cuestionarios.

Pregunta 20 (ES): ¿La relación entre esfuerzo invertido y objetivos alcanzados le ha parecido adecuada?

El grado de satisfacción entre esfuerzo y resultados alcanzados tiene un porcentaje alto, en el que 2.534 personas que corresponden al 84\%, están de acuerdo. Mientras, 488 correspondientes al $16 \%$ responden que no están de acuerdo con los resultados obtenidos.

Pregunta 22 (ES): ¿Ha tenido algún tipo de problema a la hora de presentar un trabajo o un quiz, por este medio? 
En cuanto a presentar tareas, 1.713 personas, correspondientes al 57\%, dicen no haber tenido inconvenientes; mientras que 1.279 personas, que corresponden al 43\%, dicen haber tenido problemas al realizar estas actividades.

\section{Discusión}

El resultado más interesante de la encuesta realizada fue el número de docentes que había participado en cursos MOOC: pese a pertenecer en su mayoría al sector rural, un 51,5\% afirmó haber participado en un curso masivo, lo cual corresponde a 189 respuestas de un total de 367 . Es un resultado que se iba en contra de todo pronóstico y no cabe duda que el acceso a Internet y a las TIC mejora día a día, dándonos una pauta que ha futuro la capacitación mediante cursos MOOC van a ser protagonistas y un complemento para las clases tradicionales.

En el ámbito de los cursos MOOC, las cifras de deserción son alarmantes "ya que a menudo son superiores al 90\%" (MOOC-Maker, 2016). Pero esto se puede mejorar notablemente, siempre y cuando las condiciones sean las adecuadas.

En el estudio realizado por la Universidad de Pennsylvania durante los años 2012 y 2013, solo un $4 \%$ de los estudiantes que empezaron concluyeron los cursos (Stein, 2013). Por otro lado, Katy Jordan en 2015 nos dice que el 15\% de los que empezaron concluyó el curso. A medida que el tiempo transcurre, se puede ver que más estudiantes terminan cursos MOOC, señalándonos que a futuro disminuirá el número de deserciones considerablemente.

Las principales razones por las que usuarios abandonan cursos MOOC son la mala organización del curso, la barrera tecnológica y el escaso tiempo (Leony, Muñoz-Merino, Ruipérez-Valiente, Pardo, \& Delgado, 2015). Si se toman decisiones a tiempo, se puede disminuir de manera notable el índice de deserción de los cursos MOOC.

Un $82 \%$ de los participantes mencionó que realizó el curso para aplicar en la actividad como profesor, lo que viene a ser una motivación más para culminar el curso. En otros se pudo ver que escribieron la posibilidad de subir de categoría: mientras más incentivos y motivaciones tenga un usuario MOOC, más posibilidades tiene de concluir el curso, ya que en la actualidad hay un sinnúmero de plataformas que oferta cursos similares y de forma gratuita, pero cuyo certificado no ofrece las mismas condiciones que en este caso.

\section{Conclusiones y recomendaciones}

\subsection{Conclusiones}

Hoy en día existen muchas plataformas MOOC, de renombre internacional por las universidades que dan los cursos y lo ofrecen de manera gratuita. Si deseamos que nuestro curso tenga buenos 
resultados a pesar de la competencia, es necesario encontrar un valor agregado o diferenciador hace que los usuarios se motiven y concluyan un curso con éxito. Como en este caso el certificado fue avalado por el Ministerio de Educación del Ecuador, este valor agregado es algo que las demás plataformas no ofrecen.

La motivación previa por parte de los usuarios es de mucha importancia para la realización del curso. Según la encuesta realizada, la mayoría empezó con una actitud positiva y esto ayudó a que los demás se contagiaran de esa actitud, salvo casos puntuales en los cuales una persona empezó a realizar comentarios negativos acerca del curso en los foros de discusión. Se tuvo que conversar internamente acerca de los inconvenientes que esta tenía y solucionarlos, de lo cual se llegó a la conclusión que el manejo de la plataforma no fue el adecuado por parte de la persona participante.

Es necesario un equipo de trabajo interdisciplinar, para realizar un buen MOOC. Como mínimo: un gestor de proyectos, un experto desarrollador de contenidos, un diseñador instruccional, un realizador audiovisual, un diseñador gráfico, un diseñador de multimedios interactivos y un administrador de plataforma. Además un corrector de estilo, un instructor que dará seguimiento al curso y dos responsables de soporte técnico: un experto en la materia y otro experto en el manejo de la plataforma. En un curso con más presupuesto se puede ampliar el nivel de expertos, con lo cual el curso mejoraría notablemente tanto en la parte gráfica como pedagógica.

Si no se dispone del presupuesto ideal, se puede optimizar mediante la utilización de herramientas de autor, aplicaciones que nos faciliten el trabajo con resultados óptimos. A su vez se pueden utilizar plantillas (para ahorrar tiempo), aunque no será $100 \%$ enfocado a las necesidades del curso.

Los MOOC reducen el ruido informativo que existe en Internet, clasificando la información y empaquetándola en un solo lugar, desarrollando propuestas atractivas para estudiantes que normalmente pagan mucho dinero por un certificado (Calderón, Ezeiza, \& Badiola, 2013). El tener acceso a información avalada por una institución y el realizar un convenio con alguna entidad que pague el curso por los estudiantes son incentivos adicionales para concluir un curso.

\subsection{Recomendaciones}

Es necesario sensibilizar, mediante correo electrónico o mensajes de texto, a los usuarios que no tienen experiencia en este tipo de cursos, que primero deben realizar el curso de "Introducción a la plataforma", el mismo que ayuda para un mejor desempeño y comprensión del funcionamiento de los componentes del curso. 
De ser necesario se debe quitar el porcentaje de calificación de cualquier componente que esté dando problemas a los usuarios y dejarlo como una actividad de aprendizaje. Por tal motivo se recomienda que sea gradual la dificultad de los ejercicios para que los usuarios, de a poco, puedan adaptarse. Esto sirve para que en futuros cursos de la secuencia, los usuarios puedan interactuar de forma correcta.

Para obtener mejores resultados en un curso MOOC, es recomendable que se busque el beneficio adicional a los tradicionales para sus participantes. Uno de ellos puede ser que el certificado sea avalado por otra institución u ofrecer un certificado impreso en lugar del digital. Esto hace que los participantes se motiven y escojan nuestra plataforma en lugar de otras que existen en Internet.

El mantener una actitud positiva, en la cual se pueda producir un ambiente agradable de trabajo, debe ser uno de los objetivos de los MOOC, similar a cuando se da una clase presencial, detectando el nivel de conocimiento con los cuales los alumnos se encuentran y manteniendo una mente abierta a los cambios. Estos factores se deben tomar en cuenta en cursos posteriores antes de iniciarse y, ya que son miles de usuarios, se debe estar atentos a cualquier actividad fuera de lo normal.

Se recomienda cambiar el contenido del curso de ser necesario, pues al principio no se sabe el nivel de conocimiento con que llega el usuario, pero con la ED y la participación en los foros es suficiente para darse cuenta.

Para usuarios que no manejan bien los recursos de la plataforma, es necesario tener la guía de uso, como material de consulta. También pueden ponerse en contacto con el equipo de soporte técnico, ya sea mediante el foro o cualquier otro medio, y resolver el inconveniente.

Debido a que el $43 \%$ han tenido problemas al realizar actividades, queda para trabajos futuros investigar que inconvenientes específicamente tuvieron y cuáles son los porcentajes en cada uno de ellos.

\section{Bibliografía}

Borrás, O., Martínez, M., \& Fidalgo, Á. (2014). Gamification in MOOC: challenges, opportunities and proposals for advancing MOOC model. Proceedings of the Second International Conference on Technological Ecosystems for Enhancing Multiculturality, 215-220.

Cabero, J., Llorente, M., \& Vázquez, A. (2014). Las Tipologías de MOOC: Su Diseño e Implicaciones Educativas. Profesorado, 13-26. 
Calderón, J., Ezeiza, A., \& Badiola, J. (2013). La falsa disrupción de los MOOC: La invasión de un modelo obsoleto. 6º Congreso Internacional de Educación Abierta y Tecnología Ikasnabar, (págs. 9-11).

Cormier, D., \& Siemens, G. (2010). The Open Course: Through the Open Door--Open Courses as Research, Learning, and Engagement. Educause Review, 45 (4), 30.

edX Inc. (18 de Julio de 2016). Quality education for everyone, everywhere. Recuperado el 4 de Septiembre de 2016, de edX: https://www.edx.org/about-us

Gomez, M., \& De la Garza, L. (2016). Research Analysis on MOOC Course Dropout and Retention Rates. Turkish Online Journal of Distance Education, 3-14.

Haywood, J., \& Macleod, H. (2014). To MOOC or not to MOOC? University decision-making and agile governance for educational innovation. Massive Open Online Courses: The MOOC Revolution, (págs. 1-14).

Jordan, K. (12 de Junio de 2015). MOOC Completion Rates: The Data. Recuperado el 22 de Julio de 2016, de Katy Jordan: http://www.katyjordan.com/MOOCproject.html

Lazo, J., \& Contreras, R. (2013). El Diseño en Ecuador: Comunidad de aprendizaje en un entorno cMOOC. Conferencias LACLO, (pág. 5).

Lazo, J., \& Contreras, R. (2015). Using MOOCs as a Research Approach and As Contributors to Local Knowledge. 5th Annual International Workshop on Higher Education.

Leony, D., Muñoz-Merino, P., Ruipérez-Valiente, J., Pardo, A., \& Delgado, C. (2015). Detection and Evaluation of Emotions in Massive Open Online Courses. Journal of Universal Computer Science, 21 (5), 638-655.

Luján-Mora, S. (6 de Junio de 2013). Los MOOCs un año después: ¿qué ha pasado?, ¿qué va a pasar? Recuperado el 22 de Julio de 2016, de YouTube: https://goo.gl/mmv281

Luján-Mora, S. (2013). De la clase magistral tradicional al MOOC: doce años de evolución de una asignatura sobre programación de aplicaciones web. Revista de Docencia Universitaria (REDU), 11, 279-300.

Mailhes, V., \& Raspa, J. (2015). MOOC: De la revolución educativa a la supervivencia. Letra. Imagen. Sonido. Ciudad Mediatizada. (14), 75-91. 
MOOC-Maker. (2016). Deserción y permanencia en entornos MOOC. Construction of Management Capacities of MOOCs in Higher Education, 1, 1-94.

Pappano, L. (2 de Noviembre de 2012). The Year of the MOOC. Recuperado el 22 de Julio de 2016, de The New York Times: https://goo.gl/1t0F8a

Pernías, P., \& Luján-Mora, S. (2013). Los MOOC: orígenes, historia y tipos. Comunicación y Pedagogía. Especial MOOC (269-270), 41-47.

Ramírez, M., \& Carrillo, A. (2016). MOOC as a viable option to energy sustainability and technological training. Proceedings of the 9th annual International Conference of Education, Research and Innovation.

Sanchez-Gordon, S., \& Luján-Mora, S. (2014). MOOCs gone wild. Proceedings of the 8th International Technology, Education and Development Conference (INTED 2014), (págs. 1449-1458).

Sanchez-Gordon, S., \& Luján-Mora, S. (2016). How Could MOOCs Become Accessible? The Case of edX and the Future of Inclusive Online Learning. Journal of Universal Computer Science, $22(1), 55-81$.

Sanchez-Gordon, S., Calle-Jimenez, T., \& Lujan-Mora, S. (2015). Relevance of MOOCs for Training of Public Sector Employees: Enrollment, Completion and Web Accessibility Challenges. 14th International Conference on Information Technology Based Higher Education and Training (ITHET 2015), 15, págs. 11-13.

Sanchez-Gordon, S., \& Luján-Mora, S. (2016). Barreras y estrategias de utilización de los MOOC. En H. P. Gómez, G. B. Alba, \& M. L. Carlos, La cultura de los MOOC para la innovación en educación superior desde contextos iberoamericanos (págs. 141-160). Madrid: Editorial Síntesis.

Stein, K. (2013). Penn GSE study shows MOOCs have relatively few active users, with only a few persisting to course end. Press release, University of Pennsylvania Graduate School of Education, 5. 